\title{
Hermite Interpolation Using Möbius Transformations of Planar Pythagorean-Hodograph Cubics
}

\author{
Sunhong Lee, Hyun Chol Lee, Mi Ran Lee, \\ Seungpil Jeong, and Gwang-Il Kim
}

Department of Mathematics and Research Institute of Natural Science, Gyeongsang National University, Jinju 660-701, Republic of Korea

Correspondence should be addressed to Gwang-Il Kim, gikim@gnu.ac.kr

Received 17 January 2012; Accepted 11 February 2012

Academic Editor: Saminathan Ponnusamy

Copyright $@ 2012$ Sunhong Lee et al. This is an open access article distributed under the Creative Commons Attribution License, which permits unrestricted use, distribution, and reproduction in any medium, provided the original work is properly cited.

\begin{abstract}
We present an algorithm for $C^{1}$ Hermite interpolation using Möbius transformations of planar polynomial Pythagoreanhodograph $(\mathrm{PH})$ cubics. In general, with $\mathrm{PH}$ cubics, we cannot solve $\mathrm{C}^{1}$ Hermite interpolation problems, since their lack of parameters makes the problems overdetermined. In this paper, we show that, for each Möbius transformation, we can introduce an extra parameter determined by the transformation, with which we can reduce them to the problems determining $\mathrm{PH}$ cubics in the complex plane $\mathbb{C}$. Möbius transformations preserve the PH property of $\mathrm{PH}$ curves and are biholomorphic. Thus the interpolants obtained by this algorithm are also $\mathrm{PH}$ and preserve the topology of PH cubics. We present a condition to be met by a Hermite dataset, in order for the corresponding interpolant to be simple or to be a loop. We demonstrate the improved stability of these new interpolants compared with PH quintics.
\end{abstract}

\section{Introduction}

Farouki and Sakkalis [1] introduced Pythagorean-hodograph (PH) curves, which are a special class of polynomial curves with a polynomial speed function. These curves have many computationally attractive features: in particular, their arc lengths and offset curves can be determined exactly. Farouki [2] reviews the abundant results on these curves obtained by many researchers. Hermite interpolation with $\mathrm{PH}$ curves is one of the main topics in this research (Farouki and Neff [3], Albrecht and Farouki [4], Jüttler [5], Jüttler and Mäurer [6], Farouki et al. [7], Pelosi et al. [8], and Šír et al. [9]).

In this paper, we solve the $C^{1}$ Hermite interpolation problem using the Möbius transformations of polynomial PH cubics in the plane. The use of Möbius transformation has been 
demonstrated in recent publications [10, 11]. In [11], Bartoň et al. used a general Möbius transformation in $\mathbb{R}^{3}$, which is defined as a composition of an arbitrary number of inversions with respect to spheres or planes. They showed that $(\mu \circ \mathbf{x})(t)$ is a rational PH curve for any general Möbius transformation $\mu\left(x_{1}, x_{2}, x_{3}\right)$, if $\mathbf{x}(t)$ is a polynomial PH space curve in $\mathbf{R}^{3}$. (The preservation of $\mathrm{PH}$ properties under transformation is first studied by Ueda [12].) They also presented an algorithm for $G^{1}$ Hermite interpolation. In this work, we use the classical Möbius transformation, a bijective linear fractional transformation in the extended complex plane $\mathbb{C}_{\infty}=\mathbb{C} \cup\{\infty\}$, that is,

$$
\Phi(z)=\frac{a z+b}{c z+d}
$$

for some complex numbers $a, b, c$, and $d$ for which $a d-b c \neq 0$ [13]. Using this transformation, we can solve the $C^{1}$ Hermite interpolation problems with $\mathrm{PH}$ cubics. In general, with $\mathrm{PH}$ cubics, we cannot solve $C^{1}$ Hermite interpolation problems, since their lack of parameters makes the problems overdetermined. But we can show that, for a $C^{1}$ Hermite interpolation problem, we are always able to obtain four interplants which are constructed by PH cubics. The Möbius transformation makes this possible, since it permits the introduction of a new extra parameter into the problem, which is to be reduced to a simple problem to determine $\mathrm{PH}$ cubics as follows: here we adapt the complex representation method [14] to solve the $C^{1}$ Hermite interpolation problem. The original problem is, for a Hermite dataset $\left(\mathbf{p}_{i}, \mathbf{p}_{f}, \mathbf{v}_{i}, \mathbf{v}_{f}\right)$, to find a polynomial $\mathrm{PH}$ curve $\mathbf{r}(t)$ and a Möbius transformation $\Phi(z)$, which satisfy

$$
(\Phi \circ \mathbf{r})(0)=\mathbf{p}_{i}, \quad(\Phi \circ \mathbf{r})(1)=\mathbf{p}_{f}, \quad(\Phi \circ \mathbf{r})^{\prime}(0)=\mathbf{v}_{i}, \quad(\Phi \circ \mathbf{r})^{\prime}(1)=\mathbf{v}_{f}
$$

Next, by an appropriate translation, rotation, and scaling of the dataset, we can arrange that $\mathbf{p}_{i}=\mathbf{0}$ and $\mathbf{p}_{f}=1$ and take a Möbius transformation

$$
\Phi(z)=\frac{\alpha z}{(\alpha-1) z+1}
$$

which fixes 0 and 1 , for some nonzero complex number $\alpha$. Then the inverse image of the $C^{1}$ Hermite dataset under a Möbius transformation $\Phi$ makes (1.2) into

$$
\mathbf{r}(0)=0, \quad \int_{0}^{1} \mathbf{r}^{\prime}(t) d t=1, \quad \mathbf{r}^{\prime}(0)=\frac{1}{\alpha} \mathbf{v}_{i}, \quad \mathbf{r}^{\prime}(1)=\alpha \mathbf{v}_{f}
$$

which are suitable forms for adapting the complex representation method (for details, see Section 4). Farouki and Neff [3] already solved the $C^{1}$ Hermite interpolation problem with PH quintics. According to (1.2), this is exactly the case in which $\mathbf{r}(t)$ is a quintic and $\Phi(z)$ is the identity, that is, $\alpha=1$. On the other hand, our work in this paper is the case just when $\Phi(z)$ is not the identity, that is, $\alpha \neq 1$. At the end of this paper, we will compare our interpolants with $\mathrm{PH}$ quintic ones for the same $C^{1}$ Hermite dataset.

The interpolants obtained by our method are specific rational curves represented by complex rational functions. For planar rational curves, there already exists a general theory, which were introduced by Pottmann [15] and Fiorot and Gensane [16]: they studied rational 
plane curves with rational offets. These curves are represented in the dual form, in which curves are specified using line coordinates instead of point coordinates. Pottmann showed how to design rational PH curves segments by $G^{1}$ and $G^{2}$ Hermite interpolations $[17,18]$. However, in our work, what we need is only a suitable PH cubic and a PH-preserving transformation which is algebraically simple as possible and which can generate an extraparameter, and the latter is completely settled by the classical Möbius transformation. Moreover, the transformation is biholomorphic. Thus it preserves the topology of the preimage curve (PH cubic). Therefore, the interpolants obtained by our method should have no cusp, although cusps are a generic feature of rational PH curves. They are simple curves or else loops. Hence, to obtain these, even avoiding the easy shortcut, there is no need to follow up the lengthy path with a far starting point. We just use the classical Möbius transformation of PH cubics, that is all.

The rest of this paper is organized as follows. In Sections 2 and 3, we review some basic properties of Möbius transformations and planar PH cubics. In Section 4, we solve the $C^{1}$ Hermite interpolation problem using the Möbius transformations of planar PH cubics. In Section 5, we present the condition on a Hermite dataset, which determine whether the corresponding Hermite interpolant has a loop, we also compare these new interpolants with $\mathrm{PH}$ quintics and show that the former have improved stability. We conclude this paper in Section 6.

\section{Möbius Transformations}

A Möbius transformation $\Phi(z)$ is a bijective linear fractional transformation in the extended complex plane $\mathbb{C}_{\infty}=\mathbb{C} \cup\{\infty\}$, that is,

$$
\Phi(z)=\frac{a z+b}{c z+d}
$$

for some complex numbers $a, b, c$, and $d$ for which $a d-b c \neq 0$ [13]. Then $\Phi(z)$ is a one-to-one correspondence on the extended complex plane $\mathbb{C}_{\infty}$ with its inverse

$$
\Phi^{-1}(z)=\frac{d z-b}{-c z+a}
$$

The derivative of $\Phi(z)$ is

$$
\Phi^{\prime}(z)=\frac{a d-b c}{(c z+d)^{2}}
$$

For any Möbius transformations $\Phi(z)$ and $\Psi(z),(\Psi \circ \Phi)(z)$ is also a Möbius transformation. Thus the set $\mathcal{M}$ of all Möbius transformations forms a group under composition.

A rational plane curve $\mathbf{r}(t)=x(t)+\sqrt{-1} y(t)$ is called a Pythagorean-hodograph $(\mathrm{PH})$ curve [1] if there exists a rational function $\sigma(t)$ such that

$$
x^{\prime}(t)^{2}+y^{\prime}(t)^{2}=\sigma(t)^{2}
$$


Lemma 2.1. Let $\Phi(z)$ be a Möbius transformation and $\mathbf{r}(t)$ be a polynomial PH curve. Then $\mathbf{s}(t)=$ $(\Phi \circ \mathbf{r})(t)$ is a rational $P H$ curve.

Proof. Since $\mathbf{s}^{\prime}(t)=\Phi^{\prime}(\mathbf{r}(t)) \mathbf{r}^{\prime}(t)$, we have

$$
\left|\mathbf{s}^{\prime}(t)\right|=\frac{|a d-b c|}{|c \mathbf{r}(t)+d|^{2}}\left|\mathbf{r}^{\prime}(t)\right|=\frac{|a d-b c|}{\operatorname{Re}(c \mathbf{r}(t)+d)^{2}+\operatorname{Im}(c \mathbf{r}(t)+d)^{2}}\left|\mathbf{r}^{\prime}(t)\right| .
$$

This completes the proof.

Lemma 2.1 means that Möbius transformations preserve the PH property, which is a special case of the result of Barton et al. [11].

For a polynomial PH curve $\mathbf{r}(t)$ of degree $n$, a Möbius transformation of $\mathbf{r}(t)$

$$
(\Phi \circ \mathbf{r})(t)=\frac{a \mathbf{r}(t)+b}{c \mathbf{r}(t)+d}
$$

is a rational curve, also of degree $n$, with coefficients in the complex plane $\mathbb{C}$. However, if we associate the complex plane $\mathbb{C}$ with $\mathbb{R}^{2}$, and express $(\Phi \circ \mathbf{r})(t)$ as a rational curve with real coefficients in $\mathbb{R}^{2}$ then the result is generally a rational PH curve of degree $2 n$ or $n$. If we perform a further Möbius transformation $\Psi(z)$, the rational curve $(\Psi \circ(\Phi \circ \mathbf{r}))(t)=((\Psi \circ \Phi) \circ \mathbf{r})(t)$ retains a degree of $2 n$ or $n$, since $(\Psi \circ \Phi)(z)$ is a Möbius transformation.

Lemma 2.2. Let $(\Phi \circ \mathbf{r})(t)$ be a Möbius transformation $\Phi(z)$ of a polynomial curve $\mathbf{r}(t)$, such that $(\Phi \circ \mathbf{r})(0)=0$ and $(\Phi \circ \mathbf{r})(1)=1$. Then there exist a polynomial curve $\mathbf{s}(t)$ and a Möbius transformation $\Psi(z)$, such that $\mathbf{s}(0)=0, \mathbf{s}(1)=1$, and $(\Phi \circ \mathbf{r})(t)=(\Psi \circ \mathbf{s})(t)$.

Proof. We can find a Möbius transformation $\Phi_{1}(z)=a z+b$ for some complex constants $a$ and $b$ such that $a \neq 0$ and $\mathbf{s}(t)=\left(\Phi_{1} \circ \mathbf{r}\right)(t)$ is a polynomial curve with $\mathbf{s}(0)=0$ and $\mathbf{s}(1)=1$. Consequently, we can obtain the Möbius transformation $\Psi(z)=\left(\Phi \circ \Phi_{1}^{-1}\right)(z)$ such that $(\Phi \circ \mathbf{r})(t)=$ $(\Psi \circ \mathbf{s})(t)$.

A Möbius transformation $\Psi(z)$ of this sort also fixes 0 and 1 .

Lemma 2.3. Let $\Phi(z)$ be a Möbius transformation with $\Phi(0)=0$ and $\Phi(1)=1$. Then there exists a nonzero complex constant $\alpha$ such that

$$
\Phi(z)=\frac{\alpha z}{(\alpha-1) z+1}
$$

If $\tau=|\alpha|$ and $\eta=\arg (\alpha)$, then $\Phi(z)=\left(\Phi_{\tau} \circ \Phi_{\eta}\right)(z)=\left(\Phi_{\eta} \circ \Phi_{\tau}\right)(z)$, where

$$
\Phi_{\tau}(z)=\frac{\tau z}{(\tau-1) z+1}, \quad \Phi_{\eta}(z)=\frac{e^{i \eta} z}{\left(e^{i \eta}-1\right) z+1} .
$$

Proof. Let $\Psi(z)=(a z+b) /(c z+d)$ be a Möbius transformation with $\Psi(0)=0$ and $\Psi(\infty)=\infty$. Then from $\Psi(0)=0$ we get $b=0$, and from $\Psi(\infty)=\infty$ we get $c=0$. Thus $\Psi(z)=\alpha z$, where $\alpha=a / d$. Let $\tau=|\alpha|$ and $\eta=\arg (\alpha)$. Then we obtain $\Psi(z)=\left(\Psi_{\tau} \circ \Psi_{\eta}\right)(z)=\left(\Psi_{\eta} \circ \Psi_{\tau}\right)(z)$, where $\Psi_{\tau}(z)=\tau z$ and $\Psi_{\eta}(z)=e^{i \eta} z$. 
Now let $\Phi(z)$ be a Möbius transformation with $\Phi(0)=0$ and $\Phi(1)=1$. Let $S(z)=$ $z /(z-1)$, so that $S(0)=0$ and $S(\infty)=1$. Then, since $\left(S^{-1} \circ \Phi \circ S\right)(0)=0$ and $\left(S^{-1} \circ \Phi \circ S\right)(\infty)=$ $\infty$, we get $\left(S^{-1} \circ \Phi \circ S\right)(z)=\Psi(z)$, where $\Psi(z)=\alpha z$ for some nonzero $\alpha$. Thus we obtain

$$
\Phi(z)=\left(S \circ \Psi \circ S^{-1}\right)(z)=\frac{\alpha z}{(\alpha-1) z+1} .
$$

Moreover, in the same way, we can obtain $\Phi_{\tau}(z)=\left(S \circ \Psi_{\tau} \circ S^{-1}\right)(z)$ and $\Phi_{\eta}(z)=\left(S \circ \Psi_{\eta} \circ\right.$ $\left.S^{-1}\right)(z)$, so that $\Phi(z)=\left(\Phi_{\tau} \circ \Phi_{\eta}\right)(z)=\left(\Phi_{\eta} \circ \Phi_{\tau}\right)(z)$.

$$
\text { Since } \Phi^{\prime}(z)=\alpha /((\alpha-1) z+1)^{2} \text {, we have } \Phi^{\prime}(0)=\alpha \text { and } \Phi^{\prime}(1)=1 / \alpha \text {. }
$$

\section{Planar Pythagorean-Hodograph Cubics}

A planar polynomial curve $\mathbf{r}(t)=x(t)+\sqrt{-1} y(t)$ is a PH curve [19] if and only if there exist polynomials $h(t), u(t)$, and $v(t)$, which satisfy

$$
x^{\prime}(t)=h(t)\left[u(t)^{2}-v(t)^{2}\right], \quad y^{\prime}(t)=h(t)[2 u(t) v(t)]
$$

Note that, if $\operatorname{gcd}(u(t), v(t))=1$, then $\operatorname{gcd}\left(u(t)^{2}-v(t)^{2}, 2 u(t) v(t)\right)=1$. In this paper, we will assume that $h(t)$ is monic, meaning that its leading coefficient is 1 .

A polynomial curve $\mathbf{r}(t)$ is a PH curve [14] if and only if there exists a polynomial $h(t)$ and a polynomial curve $\mathbf{w}(t)$ such that

$$
\mathbf{r}^{\prime}(t)=h(t) \mathbf{w}(t)^{2}
$$

Suppose that the PH cubic $\mathbf{r}(t)$ is a line. Then the hodograph $\mathbf{r}^{\prime}(t)$ can be expressed as $h(t)\left(x_{0}+\right.$ $\left.\sqrt{-1} y_{0}\right)$, where $x_{0}+\sqrt{-1} y_{0}$ is a nonzero point and $h(t)$ is the quadratic monic polynomial

$$
h(t)=h_{0}(1-t)^{2}+h_{1} 2(1-t) t+h_{2} t^{2}
$$

and $h_{0}, h_{1}$, and $h_{2}$ are real constants such that $h_{0}+h_{2} \neq 2 h_{1}$.

Let $\mathbf{r}(t)$ be a PH cubic for which $\mathbf{r}^{\prime}(t)=\mathbf{w}(t)^{2}$. Since $\mathbf{w}(t)$ is linear, we can write $\mathbf{w}(t)$ in Bernstein form:

$$
\mathbf{w}(t)=\mathbf{w}_{0}(1-t)+\mathbf{w}_{1} t
$$

where $\mathbf{w}_{0}$ and $\mathbf{w}_{1}$ are distinct complex constants. The hodograph $\mathbf{r}^{\prime}(t)$ can then be expressed as

$$
\mathbf{r}^{\prime}(t)=\mathbf{w}_{0}^{2}(1-t)^{2}+\mathbf{w}_{0} \mathbf{w}_{1} 2(1-t) t+\mathbf{w}_{1}^{2} t^{2}
$$

If we represent the $\mathrm{PH}$ cubic $\mathbf{r}(t)$ in the Bernstein form

$$
\mathbf{r}(t)=\mathbf{p}_{0}(1-t)^{3}+\mathbf{p}_{1} 3(1-t)^{2} t+\mathbf{p}_{2} 3(1-t) t^{2}+\mathbf{p}_{3} t^{3}
$$


then we obtain

$$
\mathbf{p}_{1}=\mathbf{p}_{0}+\frac{1}{3} \mathbf{w}_{0}^{2}, \quad \mathbf{p}_{2}=\mathbf{p}_{1}+\frac{1}{3} \mathbf{w}_{0} \mathbf{w}_{1}, \quad \mathbf{p}_{3}=\mathbf{p}_{2}+\frac{1}{3} \mathbf{w}_{1}^{2}
$$

where $\mathbf{p}_{0}$ can be chosen arbitrarily.

\section{First-Order Hermite Interpolation}

We will now solve the $C^{1}$ Hermite interpolation problem using Möbius transformations of PH cubics.

Let $\mathbf{p}_{i}$ and $\mathbf{p}_{f}$ be the initial and final points to be interpolated, where $\mathbf{p}_{i} \neq \mathbf{p}_{f}$. Let $\mathbf{v}_{i}=$ $r_{i} e^{\sqrt{-1} \theta_{i}}$ and $\mathbf{v}_{f}=r_{f} e^{\sqrt{-1} \theta_{f}}$, respectively, be the initial vector at $\mathbf{p}_{i}$ and the final vector at $\mathbf{p}_{f}$, where $r_{i}>0$ and $r_{f}>0$. For this Hermite dateset $\left(\mathbf{p}_{i}, \mathbf{p}_{f}, \mathbf{v}_{i}, \mathbf{v}_{f}\right)$, we want to find planar PH cubics $\mathbf{r}(t)$ and Möbius transformations $\Phi(z)$ which satisfy (1.2), which are equivalent to

$$
\begin{gathered}
(\Phi \circ \mathbf{r})(0)=\mathbf{p}_{i}, \quad \int_{0}^{1}(\Phi \circ \mathbf{r})^{\prime}(t) d t=\mathbf{p}_{f}-\mathbf{p}_{i} \\
(\Phi \circ \mathbf{r})^{\prime}(0)=\mathbf{v}_{i}, \quad(\Phi \circ \mathbf{r})^{\prime}(1)=\mathbf{v}_{f} .
\end{gathered}
$$

By an appropriate translation, rotation, and scaling of the data-set, we can arrange that $\mathbf{p}_{i}=\mathbf{0}$ and $\mathbf{p}_{f}=1$. Then, from Lemmas 2.2 and 2.3, we seek some nonzero constants $\alpha$ and PH cubics $\mathbf{r}(t)$, which satisfy (1.4).

4.1. Case of $r^{\prime}(t)=h(t)=h_{0}(1-t)^{2}+h_{1} 2(1-t) t+h_{2} t^{2}$

In this case, (4.1) become

$$
\mathbf{r}(0)=0, \quad h_{0}+h_{1}+h_{2}=3, \quad \alpha h_{0}=\mathbf{v}_{i}, \quad \frac{h_{2}}{\alpha}=\mathbf{v}_{f}
$$

From the second and third of these equations, we can see that Hermite interpolants $\mathbf{r}(t)$ exist if and only if $\theta_{i}+\theta_{f}=m \pi$ for some integers $m$. In this case, for $\alpha=\tau e^{\sqrt{-1} \theta_{i}}$ or $\alpha=\tau e^{\sqrt{-1}\left(\theta_{i}+\pi\right)}$, where $\tau$ is any positive number, we have

$$
h_{0}=\frac{\mathbf{v}_{i}}{\alpha}, \quad h_{2}=\alpha \mathbf{v}_{f}, \quad h_{1}=3-h_{0}-h_{2}
$$

Consequently, we can obtain the PH cubics

$$
\mathbf{r}(t)=\frac{h_{0}}{3} 3(1-t)^{2} t+\frac{h_{0}+h_{1}}{3} 3(1-t) t^{2}+\frac{h_{0}+h_{1}+h_{2}}{3} t^{3}
$$


and their Möbius transformations

$$
(\Phi \circ \mathbf{r})(t)=\frac{\alpha \mathbf{r}(t)}{(\alpha-1) \mathbf{r}(t)+1}
$$

4.2. Case of $r^{\prime}(t)=w(t)^{2}$ Where $w(t)=w_{0}(1-t)+w_{1} t$

From (3.5) and (3.6), (4.1) become

$$
\mathbf{r}(0)=0, \quad \mathbf{w}_{0}^{2}+\mathbf{w}_{0} \mathbf{w}_{1}+\mathbf{w}_{1}^{2}=3, \quad \alpha \mathbf{w}_{0}^{2}=\mathbf{v}_{i}, \quad \frac{\mathbf{w}_{1}^{2}}{\alpha}=\mathbf{v}_{f} .
$$

If we let

$$
\mathbf{v}=\frac{\sqrt{r_{i} r_{f}}}{3} e^{\sqrt{-1}\left(\theta_{i}+\theta_{f}\right) / 2}
$$

and also let $\mathbf{a}=\mathbf{w}_{0}^{2} / 3, \mathbf{b}=\mathbf{w}_{1}^{2} / 3$ and $\mathbf{k}=\mathbf{w}_{0} \mathbf{w}_{1} / 3$, then second and third equations in (4.6) imply that $\mathbf{k}=\mathbf{v}$ or $\mathbf{k}=-\mathbf{v}$, and so we have

$$
\mathbf{a b}=\mathbf{k}^{2}, \quad \mathbf{a}+\mathbf{b}=1-\mathbf{k} .
$$

Now let

$$
\begin{aligned}
& \mathbf{a}_{m}=\frac{1-\mathbf{k}+\sqrt{(1+\mathbf{k})(1-3 \mathbf{k})}}{2} \\
& \mathbf{b}_{m}=\frac{1-\mathbf{k}-\sqrt{(1+\mathbf{k})(1-3 \mathbf{k})}}{2}
\end{aligned}
$$

where $m=1$ if $\mathbf{k}=\mathbf{v}$, and $m=-1$ if $\mathbf{k}=-\mathbf{v}$. Then we have $\mathbf{a}=\mathbf{a}_{m}$ and $\mathbf{b}=\mathbf{b}_{m}$, or $\mathbf{a}=\mathbf{b}_{m}$ and $\mathbf{b}=\mathbf{a}_{m}$. Consequently, we can obtain the four PH cubics

$$
\begin{aligned}
& \mathbf{r}_{m, 1}(t)=\mathbf{a}_{m} 3(1-t)^{2} t+\left(\mathbf{a}_{m}+\mathbf{k}\right) 3(1-t) t^{2}+\left(\mathbf{a}_{m}+\mathbf{k}+\mathbf{b}_{m}\right) t^{3} \\
& \mathbf{r}_{m, 2}(t)=\mathbf{b}_{m} 3(1-t)^{2} t+\left(\mathbf{b}_{m}+\mathbf{k}\right) 3(1-t) t^{2}+\left(\mathbf{b}_{m}+\mathbf{k}+\mathbf{a}_{m}\right) t^{3}
\end{aligned}
$$

where $\mathbf{k}=\mathbf{v}$ or $\mathbf{k}=-\mathbf{v}$. Note that $\mathbf{r}_{m, 1}=\mathbf{r}_{m, 2}$ if and only if $\mathbf{k}$ is -1 or $1 / 3$. From the PH cubics $\mathbf{r}_{m, j}(t)$ we can obtain the Möbius transformations of the PH cubics $\Phi_{m, j}\left(\mathbf{r}_{m, j}(t)\right)(m=1,-1$, and $j=1,2)$, where

$$
\Phi_{m, j}(z)=\frac{\alpha_{m, j} z}{\left(\alpha_{m, j}-1\right) z+1}, \quad \alpha_{m, 1}=\frac{1}{3} \frac{\mathbf{v}_{i}}{\mathbf{a}_{m}}, \quad \alpha_{m, 2}=\frac{1}{3} \frac{\mathbf{v}_{i}}{\mathbf{b}_{m}}
$$

If $\mathbf{k}$ is nonreal, then both $\mathbf{r}_{m, 1}(t)$ and $\mathbf{r}_{m, 2}(t)$ are nonlinear. But if $\mathbf{k}$ is a real number, then $-1 \leq$ $\mathbf{k} \leq 1 / 3$ if and only if both $\mathbf{r}_{m, 1}(t)$ and $\mathbf{r}_{m, 2}(t)$ are linear.

We can summarize these results. 
Theorem 4.1. Let $\left(0,1, \mathbf{v}_{i}=r_{i} e^{\sqrt{-1} \theta_{i}}, \mathbf{v}_{f}=r_{f} e^{\sqrt{-1} \theta_{f}}\right)$ be a $C^{1}$ Hermite data-set such that $r_{i}>0$ and $r_{f}>0$.

(a) Let $\mathbf{v}$ be the vector given by (4.7), and let $\mathbf{k}$ be $\mathbf{v}$ or $-\mathbf{v}$. Then all $C^{1}$ Hermite interpolants using Möbius transformations of planar PH cubics $\mathbf{r}(t)$, such that $\mathbf{r}^{\prime}(t)=\mathbf{w}(t)^{2}$ for some linear curve $\mathbf{w}(t)$, are $\Phi_{m, j}\left(\mathbf{r}_{m, j}(t)\right)(m=1,-1$, and $j=1,2)$, from (4.10) and (4.11), where $\mathbf{a}_{m}$ and $\mathbf{b}_{m}$ are given by (4.9).

(b) $C^{1}$ Hermite interpolants using Möbius transformations of planar PH cubics $\mathbf{r}(t)$, such that $\mathbf{r}^{\prime}(t)=h_{0}(1-t)^{2}+h_{1} 2(1-t) t+h_{2} t^{2}$ for some real number $h_{0}, h_{1}$, and $h_{2}$ such that $h_{0}+$ $h_{2} \neq h_{1}$, exist if and only if $\theta_{i}+\theta_{f}=m \pi$ for some integers $m$. In this case, the interpolants $(\Phi \circ \mathbf{r})(t)$ are given by (4.5), where $\mathbf{r}(t)$ is given by (4.3) and (4.4), where $\alpha=\tau e^{\sqrt{-1} \theta_{i}}$ or $\alpha=\tau e^{\sqrt{-1}\left(\theta_{i}+\pi\right)}$ for any positive number $\tau$.

\section{Best Interpolant}

In this section we consider how to choose the best interpolant for a given Hermite data-set $\left(\mathbf{p}_{i}=\mathbf{0}, \mathbf{p}_{f}=1, \mathbf{v}_{i}, \mathbf{v}_{f}\right)$.

We will begin by presenting a condition under which the Möbius transformation of a PH cubic $(\Phi \circ \mathbf{r})(t)$ has a loop, where $\mathbf{r}^{\prime}(t)=\left(\mathbf{w}_{0}(1-t)+\mathbf{w}_{1} t\right)^{2}$ for some distinct complex constants $\mathbf{w}_{0}$ and $\mathbf{w}_{1}$. Since $\Phi(z)$ represents a one-to-one correspondence on the extended complex plane, the condition that $\mathbf{r}(t)$ has a loop is both necessary and sufficient to establish that $(\Phi \circ \mathbf{r})(t)$ has a loop. Under the conditions $\mathbf{r}(0)=0$ and $\mathbf{r}(1)=1$, the PH cubic $\mathbf{r}(t)$ is given by $\mathbf{r}(t)=A(t-B)^{3}+C$, where

$$
A=\frac{\left(\mathbf{w}_{0}-\mathbf{w}_{1}\right)^{2}}{3}, \quad B=\frac{\mathbf{w}_{0}}{\mathbf{w}_{0}-\mathbf{w}_{1}}, \quad C=\frac{\mathbf{w}_{0}^{3}}{3\left(\mathbf{w}_{0}-\mathbf{w}_{1}\right)} .
$$

The condition that there exist constants $t_{1}$ and $t_{2}$, such that $0 \leq t_{1}<t_{2} \leq 1$ and $\mathbf{r}\left(t_{1}\right)=\mathbf{r}\left(t_{2}\right)$, is necessary and sufficient to establish that $\mathbf{r}(t)$ has a loop. From $\mathbf{r}\left(t_{1}\right)=\mathbf{r}\left(t_{2}\right)$, we can obtain $3 B^{2}-3\left(t_{1}+t_{2}\right) B+\left(t_{1}^{2}+t_{1} t_{2}+t_{2}^{2}\right)=0$, which implies

$$
B=\frac{3\left(t_{1}+t_{2}\right) \pm \sqrt{-1}\left(t_{2}-t_{1}\right) \sqrt{3}}{6} .
$$

This equation is equivalent to

$$
t_{1}+t_{2}=2 \operatorname{Re} B, \quad t_{2}-t_{1}=2 \sqrt{3}|\operatorname{Im} B|,
$$

and hence

$$
t_{1}=\operatorname{Re} B-\sqrt{3}|\operatorname{Im} B|, \quad t_{2}=\operatorname{Re} B+\sqrt{3}|\operatorname{Im} B| .
$$

Consequently, $\mathbf{r}(t)$ has a loop if and only if $B \in \Omega_{1} \cup \Omega_{2}$, (see Figure 1) where

$$
\begin{aligned}
& \Omega_{1}=\{z \in \mathbb{C} 0 \leq \operatorname{Re} z-\sqrt{3} \operatorname{Im} z<\operatorname{Re} z+\sqrt{3} \operatorname{Im} z \leq 1\}, \\
& \Omega_{2}=\{z \in \mathbb{C} \mid 0 \leq \operatorname{Re} z+\sqrt{3} \operatorname{Im} z<\operatorname{Re} z-\sqrt{3} \operatorname{Im} z \leq 1\} .
\end{aligned}
$$




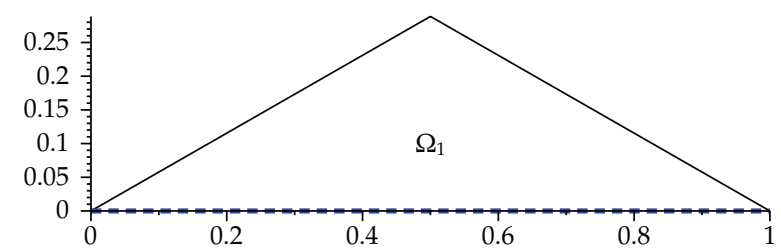

(a)

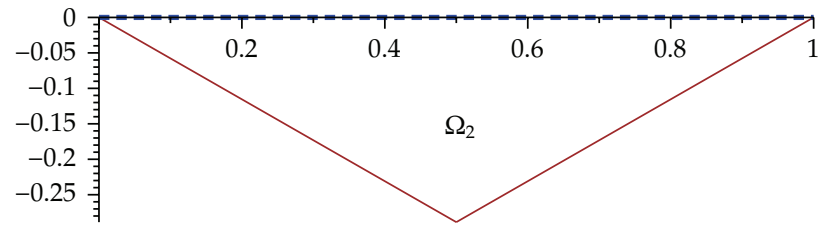

(b)

Figure 1: Areas of $\Omega_{1}$ and $\Omega_{2}: B$ belongs to $\Omega_{1} \cup \Omega_{2}$ if and only if $\mathbf{r}(t)$ has a loop.

On the other hand, the PH cubic $\mathbf{r}(t)$ can be represented by

$$
\mathbf{r}(t)=\mathbf{a} 3(1-t)^{2} t+(\mathbf{a}+\mathbf{k}) 3(1-t) t^{2}+(\mathbf{a}+\mathbf{k}+\mathbf{b}) t^{3},
$$

where $\mathbf{a}=\mathbf{w}_{0}^{2} / 3, \mathbf{k}=\mathbf{w}_{0} \mathbf{w}_{1} / 3$, and $\mathbf{b}=\mathbf{w}_{1}^{2} / 3$. From $\mathbf{k}=\mathbf{w}_{0} \mathbf{w}_{1} / 3$ and $B=\mathbf{w}_{0} /\left(\mathbf{w}_{0}-\mathbf{w}_{1}\right)$, we can obtain

$$
B^{2}-B=\frac{\mathbf{k}}{1-3 \mathbf{k}}=\frac{1}{1 / \mathbf{k}-3}
$$

and hence

$$
\mathbf{k}=\frac{1}{3}-\frac{1}{3\left(3 B^{2}-3 B+1\right)}
$$

Note that

$$
\begin{aligned}
&\left\{\frac{1}{3}-\right.\left.\frac{1}{3\left(3 \mathbf{z}^{2}-3 \mathbf{z}+1\right)} \in \mathbb{C} \mid 0 \leq \operatorname{Re} \mathbf{z}-\sqrt{3} \operatorname{Im} \mathbf{z}<\operatorname{Re} \mathbf{z}+\sqrt{3} \operatorname{Im} \mathbf{z} \leq 1\right\} \\
&=\left\{\frac{1}{3}-\frac{1}{3\left(3 \mathbf{z}^{2}-3 \mathbf{z}+1\right)} \in \mathbb{C} \mid 0 \leq \operatorname{Re} \mathbf{z}+\sqrt{3} \operatorname{Im} \mathbf{z}<\operatorname{Re} \mathbf{z}-\sqrt{3} \operatorname{Im} \mathbf{z} \leq 1\right\} .
\end{aligned}
$$

Therefore we conclude as following.

Theorem 5.1. Suppose that $(\Phi \circ \mathbf{r})(t)$ is a Möbius transformation of a planar PH cubic, such that $\mathbf{r}(0)=0, \mathbf{r}(1)=1$, and $\mathbf{r}^{\prime}(t)=\left(\mathbf{w}_{0}(1-t)+\mathbf{w}_{1} t\right)^{2}$ for some distinct complex constants $\mathbf{w}_{0}$ and $\mathbf{w}_{1}$ (see Figure 2$)$. Then $(\Phi \circ \mathbf{r})(t)(0 \leq t \leq 1)$ is a simple curve if and only if $\mathbf{w}_{0} \mathbf{w}_{1} / 3 \notin D$, where

$$
D=\left\{\frac{1}{3}-\frac{1}{3\left(3 \mathbf{z}^{2}-3 \mathbf{z}+1\right)} \in \mathbb{C} \mid 0 \leq \operatorname{Re} \mathbf{z}-\sqrt{3} \operatorname{Im} \mathbf{z}<\operatorname{Re} \mathbf{z}+\sqrt{3} \operatorname{Im} \mathbf{z} \leq 1\right\} .
$$




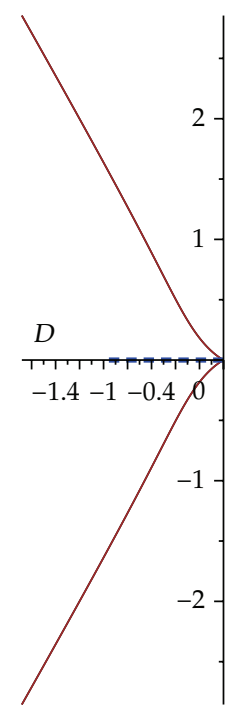

Figure 2: Area of $D: \mathbf{w}_{0} \mathbf{w}_{1} / 3 \notin D$ if and only if $(\Phi \circ \mathbf{r})(t)$ in Theorem 5.1 is a simple curve.

For a given Hermite data-set $\left(\mathbf{0}, 1, \mathbf{v}_{i}, \mathbf{v}_{f}\right)$, the term $\mathbf{v}$ in (4.7) belongs to $D$ if and only if $\left(\Phi_{1,1} \circ \mathbf{r}_{1,1}\right)(t)$ and $\left(\Phi_{1,2} \circ \mathbf{r}_{1,2}\right)(t)$ have a loop; and $-\mathbf{v}$ belongs to $D$ if and only if $\left(\Phi_{-1,1} \circ \mathbf{r}_{-1,1}\right)(t)$ and $\left(\Phi_{-1,2} \circ \mathbf{r}_{-1,2}\right)(t)$ have a loop. Note that $D$ is a subset of the left half-plane, that is, $D \subset$ $\{z \in \mathbb{Z}: \operatorname{Re} z<0\}$. Thus we can deduce that both $\left(\Phi_{1,1} \circ \mathbf{r}_{1,1}\right)(t)$ and $\left(\Phi_{1,2} \circ \mathbf{r}_{1,2}\right)(t)$, or both $\left(\Phi_{-1,1} \circ \mathbf{r}_{-1,1}\right)(t)$ and $\left(\Phi_{-1,1} \circ \mathbf{r}_{-1,2}\right)(t)$ are simple curves. From these simple curves we can choose a best interpolant, which is that with the least bending energy

$$
E((\Phi \circ \mathbf{r})(t))=\int_{\Phi \circ \mathbf{r}} \kappa^{2} d s=\int_{0}^{1} \kappa(t)^{2}\left|(\Phi \circ \mathbf{r})^{\prime}(t)\right| d t
$$

where $\mathcal{\kappa}$ is the curvature of $(\Phi \circ \mathbf{r})(t)$.

Example 5.2. Consider a Hermite data-set $\left(0,1,2 e^{-\sqrt{-1} \pi / 4}, 2 e^{-\sqrt{-1} \pi / 8}\right)$. Then the vector $\mathbf{v}$ becomes

$$
\mathbf{v}=\frac{\sqrt{r_{i} r_{f}}}{3} e^{\sqrt{-1}\left(\theta_{i}+\theta_{f}\right) / 2}=\frac{2}{3} e^{-\sqrt{-1} 3 \pi / 16}
$$

Thus $\mathbf{v} \notin D$ and $-\mathbf{v} \in D$, which implies that $\left(\Phi_{1,1} \circ \mathbf{r}_{1,1}\right)(t)$ and $\left(\Phi_{1,2} \circ \mathbf{r}_{1,2}\right)(t)$ are simple but $\left(\Phi_{-1,1} \circ \mathbf{r}_{-1,1}\right)(t)$ and $\left(\Phi_{-1,2} \circ \mathbf{r}_{-1,2}\right)(t)$ each have a loop. See Figure 3.

Example 5.3. In the case of a Hermite data-set $\left(0,1, e^{-\sqrt{-1} \pi / 5}, e^{-\sqrt{-1} \pi / 5}\right)$, the vector $\mathbf{v}$ becomes

$$
\mathbf{v}=\frac{\sqrt{r_{i} r_{f}}}{3} e^{\sqrt{-1}\left(\theta_{i}+\theta_{f}\right) / 2}=\frac{1}{3} e^{-\sqrt{-1} 2 \pi / 5} .
$$

Thus $\mathbf{v} \notin D$ and $-\mathbf{v} \notin D$, which implies that $\left(\Phi_{1,1} \circ \mathbf{r}_{1,1}\right)(t),\left(\Phi_{1,2} \circ \mathbf{r}_{1,2}\right)(t),\left(\Phi_{-1,1} \circ \mathbf{r}_{-1,1}\right)(t)$, and $\left(\Phi_{-1,2} \circ \mathbf{r}_{-1,2}\right)(t)$ are all simple. See Figure 4. 


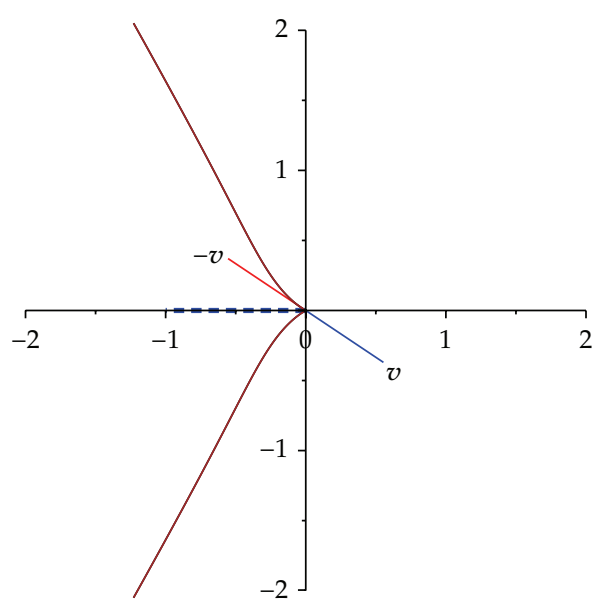

(a)

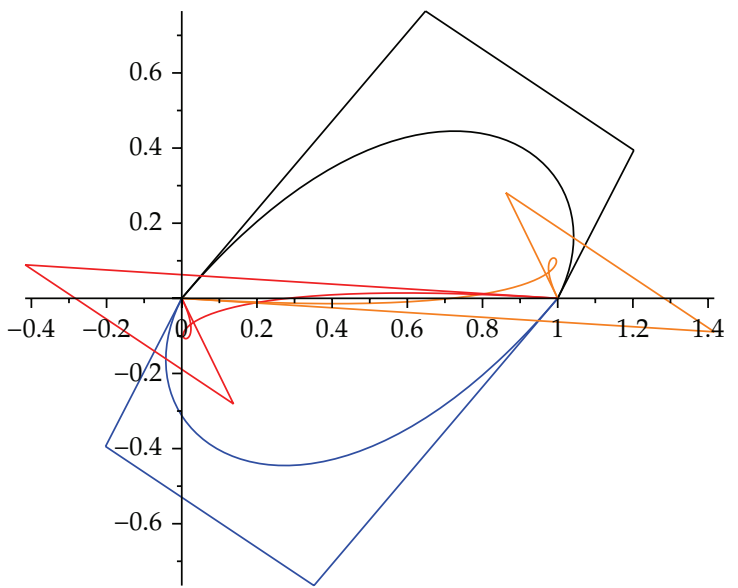

(b)

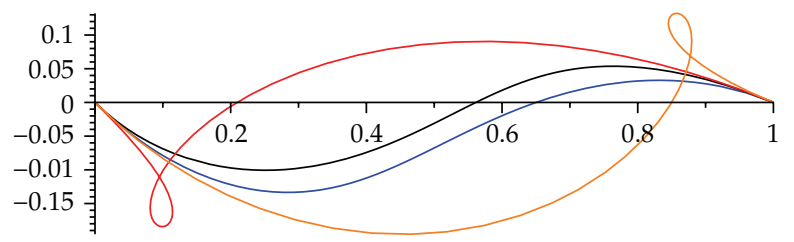

(c)

Figure 3: For the Hermite dataset $\left(0,1,2 e^{-\sqrt{-1} \pi / 4}, 2 e^{-\sqrt{-1} \pi / 8}\right)$, the graph on the left shows that $-\mathbf{v} \in D$ and $\mathbf{v} \notin D$; the central graph shows the PH cubics $\mathbf{r}(t)$ with their control polygons; the graph on the right shows the four interpolants.

Example 5.4. Consider a family of $C^{1}$ Hermite data-sets $(0,2, k(1+\sqrt{-1}), 1+2 \sqrt{-1})$, where $k=$ $1,5,10,20$. We construct $C^{1}$ Hermite interpolants that satisfy these data-sets using Möbius transformations of PH cubics, and also PH quintics, all shown in Figure 5. The Möbius transformations of the PH cubics always provide two S-shaped simple curves and two other curves; the latter are C-shaped simple curves when $k=1$ or 5 and have a single loop in the other cases. As the parametric speed of the initial Hermite condition increases, the C-shaped interpolants change from simple curves to single loops, while the simple S-shaped interpolants retain their original shape characteristics. We also observe that, unlike the S-shaped interpolants produced by Möbius transformations of PH cubics, the S-shaped PH quintic interpolants may be simple (like the curve labeled 4 in Figure 5), or have one or two loops (some PH quintics labeled 2 in Figure 5 are S-shaped double loops).

We observed the behavior of these interpolants as the parametric speed at the endpoints changes. As this speed increases, the arc-lengths of $\mathrm{PH}$ quintics increase rapidly, but the arc-length, of Möbius transformations of PH cubics are generally less affected. In particular, the simple S-shaped interpolants, produced by Möbius transformations of PH cubics show little change in arc-length. Table 1 shows that these latter interpolants have both lower bending energies and shorter arc-lengths, than all the other interpolants we are considering. If we look at Table 1 and identify the most shapely interpolants with the lowest bending energies, we find that the best Möbius transformation of a PH cubic is always S-shaped and 


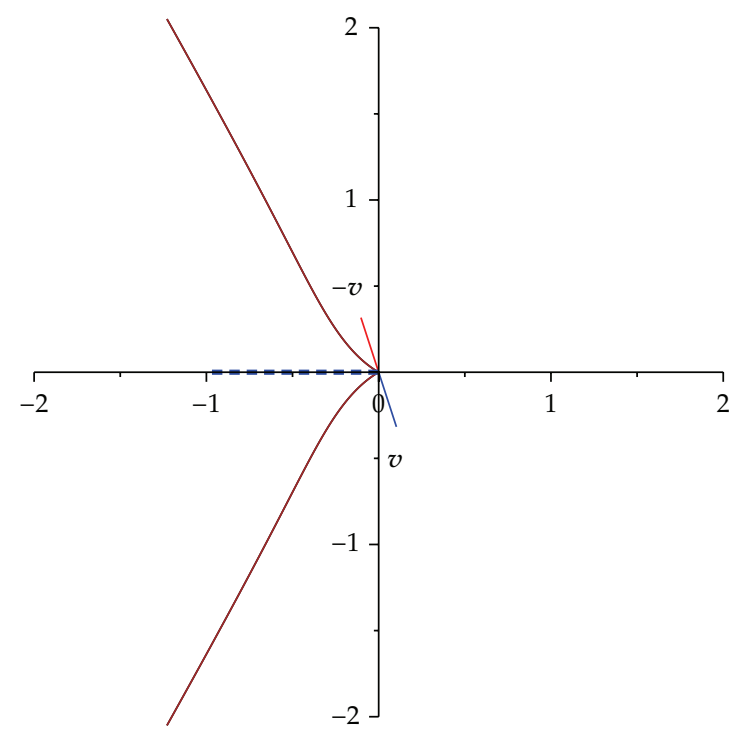

(a)

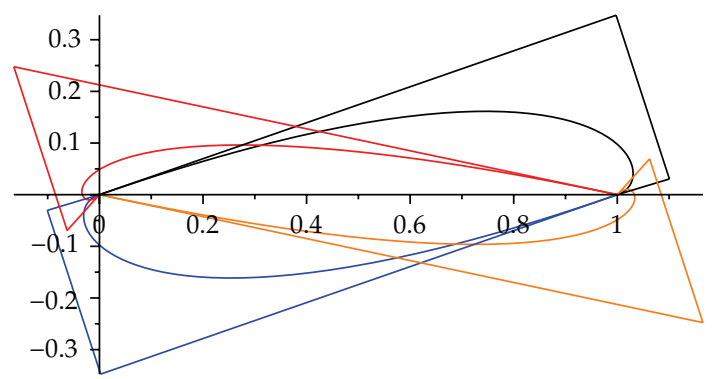

(b)

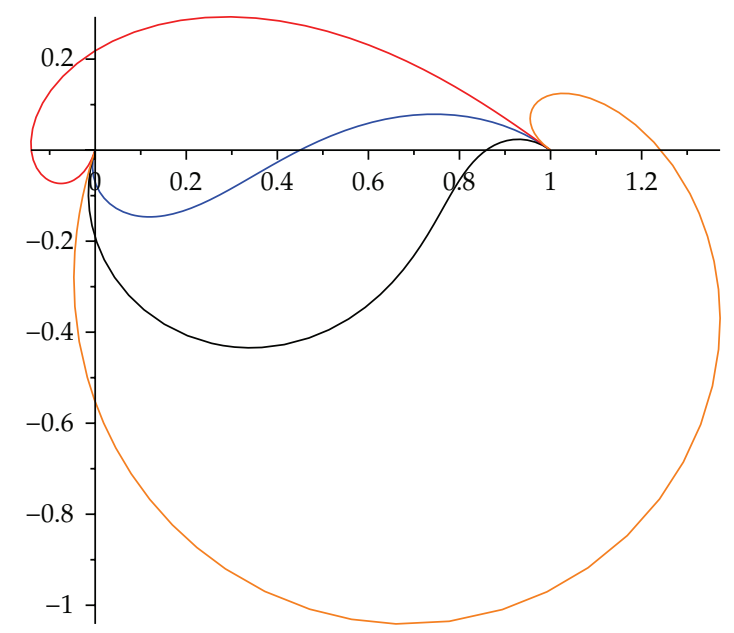

(c)

Figure 4: For the Hermite data-set $\left(0,1, e^{-\sqrt{-1} 3 \pi / 5}, e^{-\sqrt{-1} \pi / 5}\right)$, the graph on the left shows that $-\mathbf{v} \notin D$ and $\mathbf{v} \notin D$; the central graph shows the PH cubics $\mathbf{r}(t)$ with their control polygons; the graph on the right shows the four interpolants. 


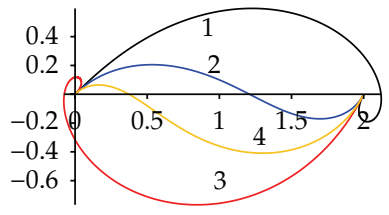

(a)

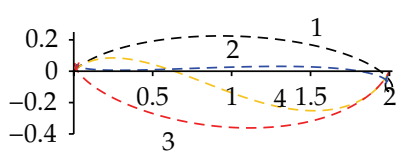

$(\mathrm{a})^{\prime}$

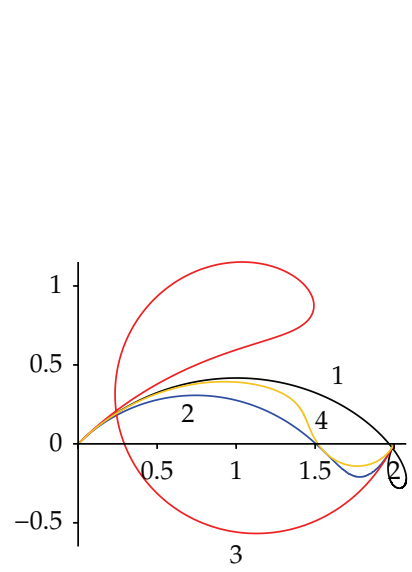

(d)

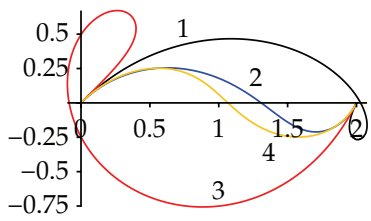

(b)

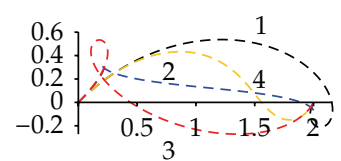

$(\mathrm{b})^{\prime}$

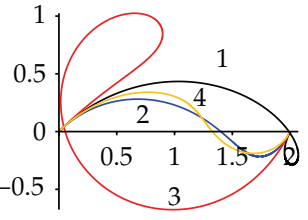

(c)

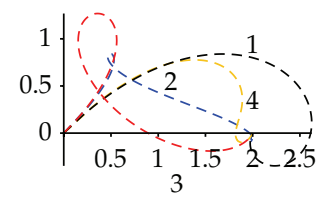

$(c)^{\prime}$

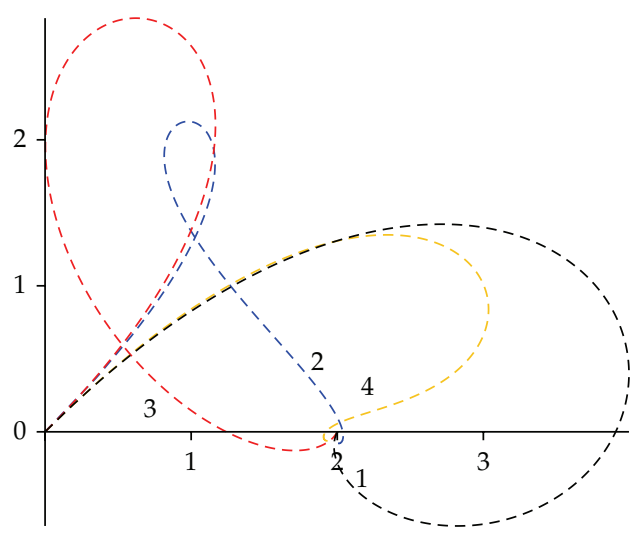

$(\mathrm{d})^{\prime}$

Figure 5: Comparison of pairs of PH interpolants, satisfying the same $C^{1}$ Hermite data-set $(0,2, k(1+\sqrt{-1})$, $1+2 \sqrt{-1}$ ), when $k=1,5,10,20$ : (a), (b), (c), and (d), respectively, show the Möbius transformations of the PH cubics $M C_{i}$ when $k=1,5,10,20$; and $(\mathrm{a})^{\prime},(\mathrm{b})^{\prime},(\mathrm{c})^{\prime}$, and $(\mathrm{d})^{\prime}$ show the correspoding PH quintics $Q_{i}$.

simple. However, the merit of the $\mathrm{PH}$ quintic interpolants depends on the parametric speeds at their end points. For example, in Figures $5(\mathrm{a})^{\prime}$ and $5(\mathrm{~b})^{\prime}$, the curves labeled 4 are best, while the curves labeled 1 are best in Figures $5(\mathrm{c})^{\prime}$ and $5(\mathrm{~d})^{\prime}$. Looking closely at the PH quintic interpolants, we see that the simple S-shaped curve with the best shape when $k=1$ becomes less and less acceptable as the parametric speeds at the end-points increase. But the interpolants labeled 1 in Figures 5(a)', 5(b), $5(\mathrm{c})^{\prime}$, and 5(d)' exhibit the opposite behavior: initially these curves are $\mathrm{C}$-shaped loops with high bending energies when $k=1$; but as the parametric speed increases, they become C-shaped simple curves with lower bending energies. When $k$ reaches 20, it has the best shape but the greatest arc-length. This suggests that the best-shaped interpolants, produced by Möbius transformations of $\mathrm{PH}$ cubics are more stable than the corresponding PH quintics, in the sense that the former largely achieve a lower arclength and bending energy than the latter, except when the end-point speeds are significantly asymmetric, as we see when $k=20$ in this example. 
Table 1: Comparison of arc-length and bending energy for the interpolants of Figure 5.

\begin{tabular}{lcccccccc}
\hline$k=1$ & $M C_{1}$ & $M C_{2}$ & $M C_{3}$ & $M C_{4}$ & $Q_{1}$ & $Q_{2}$ & $Q_{3}$ & $Q_{4}$ \\
\hline arc-length & 3.03 & 2.19 & 3.10 & 2.29 & 2.34 & 2.16 & 2.34 & 2.16 \\
BE & 45.0 & 5.5 & 72.8 & 6.8 & 149 & 3106 & 273 & 5.3 \\
\hline$k=5$ & $M C_{1}$ & $M C_{2}$ & $M C_{3}$ & $M C_{4}$ & $Q_{1}$ & $Q_{2}$ & $Q_{3}$ & $Q_{4}$ \\
\hline arc-length & 2.93 & 2.28 & 4.50 & 2.31 & 3.05 & 2.40 & 3.05 & 2.40 \\
BE & 50.2 & 6.5 & 20.9 & 5.7 & 36.1 & 762 & 47.3 & 10.0 \\
\hline$k=10$ & $M C_{1}$ & $M C_{2}$ & $M C_{3}$ & $M C_{4}$ & $Q_{1}$ & $Q_{2}$ & $Q_{3}$ & $Q_{4}$ \\
\hline arc-length & 2.89 & 2.31 & 5.47 & 2.36 & 4.42 & 3.02 & 4.42 & 3.02 \\
BE & 54.03 & 8.2 & 16.6 & 7.5 & 14.4 & 345.9 & 19.3 & 36.9 \\
\hline$k=20$ & $M C_{1}$ & $M C_{2}$ & $M C_{3}$ & $M C_{4}$ & $Q_{1}$ & $Q_{2}$ & $Q_{3}$ & $Q_{4}$ \\
\hline arc-length & 2.85 & 2.34 & 6.13 & 2.40 & 7.91 & 5.39 & 7.91 & 5.39 \\
BE & 60.1 & 11.9 & 17.7 & 11.3 & 8.0 & 136 & 10.7 & 97.9 \\
\hline
\end{tabular}

\section{Conclusions}

Möbius transformations preserve Pythagorean-hodograph properties. For any $C^{1}$ Hermite data-set, we can generally obtain four $C^{1}$ Hermite interpolants as Möbius transformations of $\mathrm{PH}$ cubics. We have proved that these interpolants are always simple curves or single loops, and that at least two of them must be simple. We have also presented the condition that an interpolant must meet if it is to be a simple curve.

We compared the shape characteristics of $C^{1}$ Hermite interpolants, produced by Möbius transformations of $\mathrm{PH}$ cubics, together with their response to changes of parametric speed at their end points, with the same data for $\mathrm{PH}$ quintic interpolants satisfying an identical $C^{1}$ Hermite dataset: we found that interpolants produced by Möbius transformations of $\mathrm{PH}$ cubics generally have lower bending energies and shorter arc-lengths than PH quintics.

One avenue for further research is to look for ways of predicting how the geometry of Möbius transformation of $\mathrm{PH}$ cubics will be determined by a particular $C^{1}$ Hermite data-set. Another avenue to explore would be the application of Möbius transformations to other interpolation problems involving $\mathrm{PH}$ (or MPH) curves, in both two and three dimensions. In particular, we might look to complete the geometric characterization of Möbius transformation of $\mathrm{PH}$ cubics in $\mathrm{C}^{1}$ Hermite interpolation.

\section{Acknowledgment}

This research was supported by the Korea Science and Engineering Foundation (KOSEF) Grant funded by the Korea government (MEST) (2009-0073488).

\section{References}

[1] R. T. Farouki and T. Sakkalis, "Pythagorean hodographs," Journal of Research and Development, vol. 34, no. 5, pp. 736-752, 1990.

[2] R. T. Farouki, Pythagorean-Hodograph Curves: Algebra and Geometry Inseparable, vol. 1 of Geometry and Computing, Springer, Berlin, Germany, 2008.

[3] R. T. Farouki and C. A. Neff, "Hermite interpolation by Pythagorean hodograph quintics," Mathematics of Computation, vol. 64, no. 212, pp. 1589-1609, 1995. 
[4] G. Albrecht and R. T. Farouki, "Construction of $C^{2}$ Pythagorean-hodograph interpolating splines by the homotopy method," Advances in Computational Mathematics, vol. 5, no. 4, pp. 417-442, 1996.

[5] B. Jüttler, "Hermite interpolation by Pythagorean hodograph curves of degree seven," Mathematics of Computation, vol. 70, no. 235, pp. 1089-1111, 2001.

[6] B. Jüttler and C. Mäurer, "Cubic Pythagorean hodograph spline curves and applications to sweep surface modeling," Computer-Aided Design, vol. 31, pp. 73-83, 1999.

[7] R. T. Farouki, M. al-Kandari, and T. Sakkalis, "Hermite interpolation by rotation-invariant spatial Pythagorean-hodograph curves," Advances in Computational Mathematics, vol. 17, no. 4, pp. 369-383, 2002.

[8] F. Pelosi, R. T. Farouki, C. Manni, and A. Sestini, "Geometric Hermite interpolation by spatial Pythagorean-hodograph cubics," Advances in Computational Mathematics, vol. 22, no. 4, pp. 325-352, 2005.

[9] Z. Šír, B. Bastl, and M. Lávička, "Hermite interpolation by hypocycloids and epicycloids with rational offsets," Computer Aided Geometric Design, vol. 27, no. 5, pp. 405-417, 2010.

[10] A. I. Kurnosenko, "Two-point $G^{2}$ Hermite interpolation with spirals by inversion of hyperbola," Computer Aided Geometric Design, vol. 27, no. 6, pp. 474-481, 2010.

[11] M. Bartoň, B. Jüttler, and W. Wang, "Construction of rational curves with rational. Rotation-minimizing frames via Möbius transformations," in Mathematical Methods for Curves and Surfaces, Lecture Notes in Computer Science, pp. 15-25, Springer, Berlin, Germany, 2010.

[12] K. Ueda, "Spherical Pythagorean-hodograph curves," in Mathematical Methods for Curves and Surfaces, II (Lillehammer, 1997), Innovations in Applied Mathematics, pp. 485-492, Vanderbilt University Press, Nashville, Tenn, USA, 1998.

[13] L. V. Ahlfors, Complex Analysis, International Series in Pure and Applied Mathematics, McGraw-Hill, New York, NY, USA, Third edition, 1978.

[14] R. T. Farouki, "The conformal map $z \rightarrow z^{2}$ of the hodograph plane," Computer Aided Geometric Design, vol. 11, no. 4, pp. 363-390, 1994.

[15] H. Pottmann, "Rational curves and surfaces with rational offsets," Computer Aided Geometric Design, vol. 12, no. 2, pp. 175-192, 1995.

[16] J.-C. Fiorot and Th. Gensane, "Characterizations of the set of rational parametric curves with rational offsets," in Curves and Surfaces in Geometric Design, P. J. Laurent, A. Le Mehaute, and L. L. Schumaker, Eds., pp. 153-160, A K Peters, Wellesley, Mass, USA, 1994.

[17] H. Pottmann, "Applications of the dual Bézier representation of rational curves and surfaces," in Curves and Surfaces in Geometric Design (Chamonix-Mont-Blanc, 1993), P. J. Laurent, A. Le Mehaute, and L. L. Schumaker, Eds., pp. 377-384, A K Peters, Wellesley, Mass, USA, 1994.

[18] H. Pottmann, "Curve design with rational Pythagorean-hodograph curves," Advances in Computational Mathematics, vol. 3, no. 1-2, pp. 147-170, 1995.

[19] K. K. Kubota, "Pythagorean triples in unique factorization domains," The American Mathematical Monthly, vol. 79, pp. 503-505, 1972. 


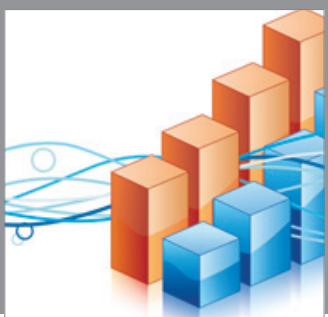

Advances in

Operations Research

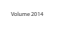

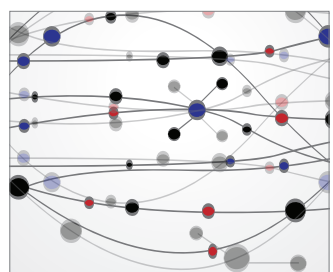

\section{The Scientific} World Journal
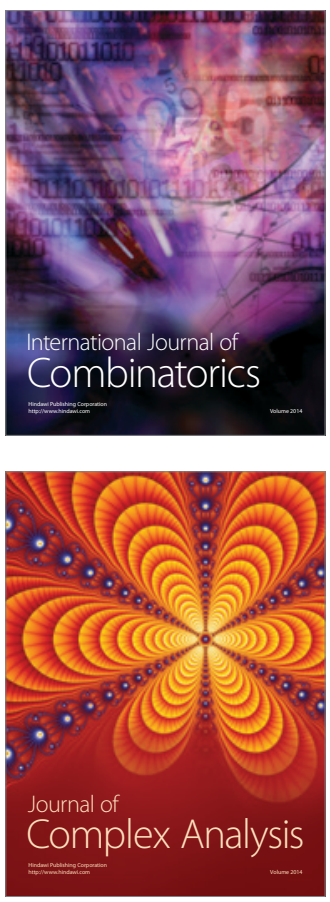

International Journal of

Mathematics and

Mathematical

Sciences
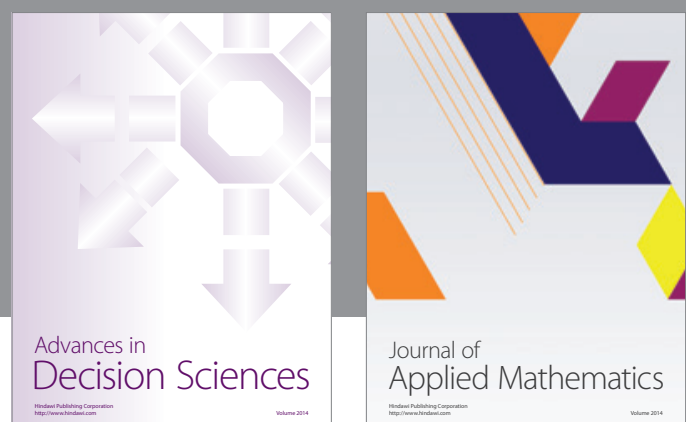

Journal of

Applied Mathematics
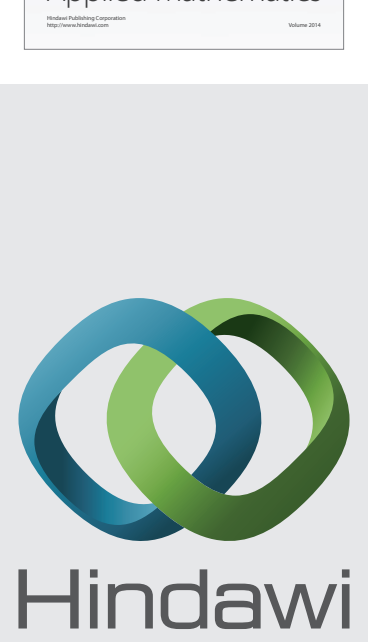

Submit your manuscripts at http://www.hindawi.com
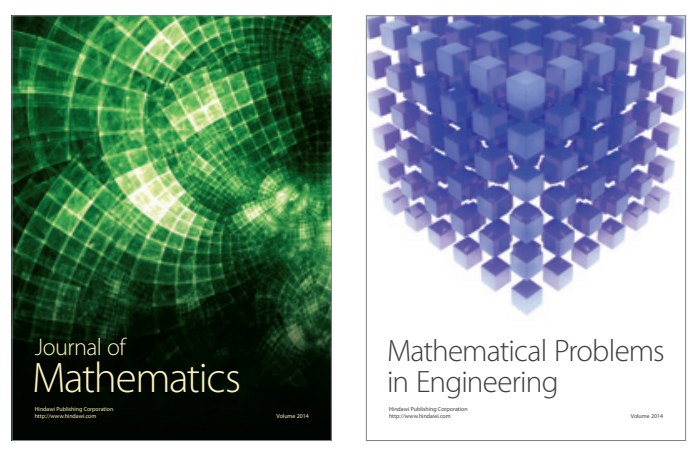

Mathematical Problems in Engineering
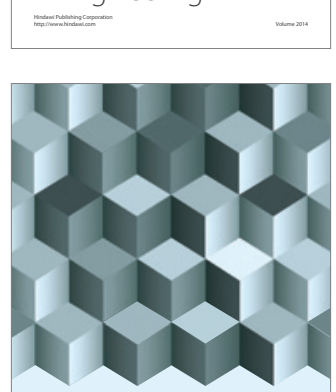

Journal of

Function Spaces
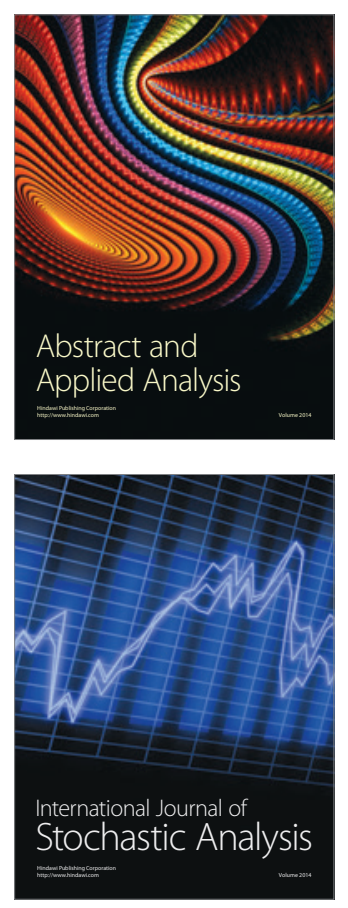

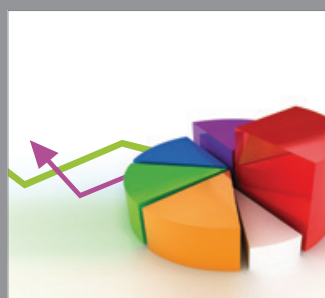

ournal of

Probability and Statistics

Promensencen
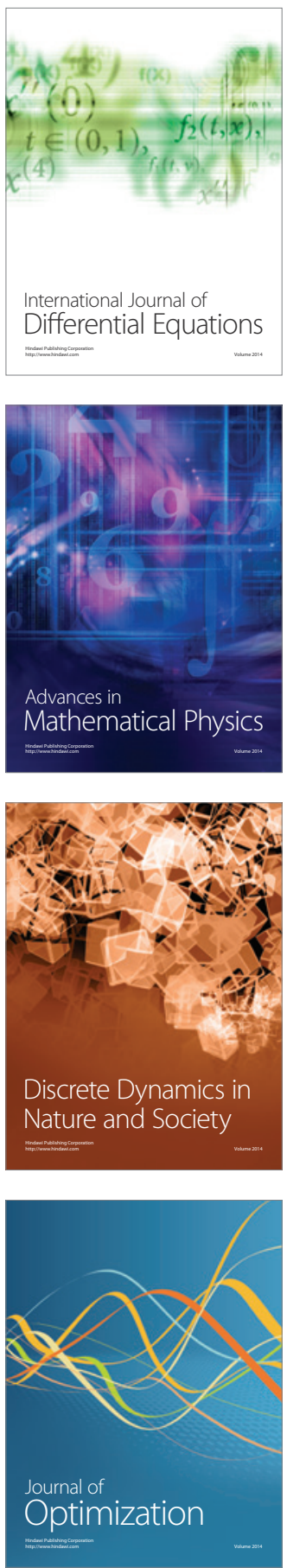